Europhys. Lett., 70 (6), pp. 810-816 (2005)

DOI: $10.1209 / \mathrm{epl} / \mathrm{i} 2005-10049-7$

\title{
Lorentz-force-induced asymmetry in the Aharonov-Bohm effect in a three-terminal semiconductor quantum ring
}

\author{
B. Szafran ${ }^{1,2}$ and F. M. Peeters ${ }^{1}$ \\ 1 Departement Fysica, Universiteit Antwerpen (Campus Middelheim) \\ Groenenborgerlaan 171, B-2020 Antwerpen, Belgium \\ 2 Faculty of Physics and Nuclear Techniques, AGH University of Science and \\ Technology - al. Mickiewicza 30, 30-059 Kraków, Poland
}

received 19 April 2005; accepted 28 April 2005

published online 18 May 2005

PACS. 73.63.-b - Electronic transport in nanoscale materials and structures.

PACS. 73.23. - b - Electronic transport in mesoscopic systems.

\begin{abstract}
Based on a solution of the time-dependent Schrödinger equation, we propose an experimental setup to measure the effect of the Lorentz force in a semiconductor AharonovBohm ring connected to one input and two symmetrically placed output leads. While in a two-terminal device the Lorentz force only leads to a decreased Aharonov-Bohm oscillation amplitude, in the two-output ring it produces a distinct imbalance in the electron transfer probability from the input to the two output leads. Depending on the value of the momentum of the incident electron locking or antilocking of the transfer probability extrema for both leads appears as a function of the magnetic field.
\end{abstract}

Introduction. - The Aharonov-Bohm [1] (AB) shift of the electron wave function by the vector potential of the magnetic field leads to oscillations of the conductance and other electric quantities measured in metal [2] and semiconductor [3-6] rings. A theory for the conductance oscillations was developed [7-10] in a strictly one-dimensional model of the ring assuming that the flux of the magnetic field through the ring is spatially separated from the electrons. However, actual experiments [3-6] are performed in homogenous magnetic fields, so that the electrons flowing through the ring are subject to the Lorentz force. The effect of the magnetic forces is well known from, e.g., electron diffraction on a biprism in which the magnetic field results in a shift of the maximum of the diffraction pattern, in accordance with the classical laws due to the Ehrenfest theorem [11]. We report here the numerical solution of the time-dependent Schrödinger equation for a single-electron transport through a GaAs quantum ring in a homogenous magnetic field. We find that the Lorentz force leads to a preferential electron injection in one of the arms of the ring, resulting in the suppression of the $\mathrm{AB}$ oscillations at high magnetic field. Although in metal rings the $\mathrm{AB}$ oscillations continue up to $8 \mathrm{~T}$, corresponding to $10^{4}$ flux quanta passing through the ring [2], in most experiments [3-5] on semiconducting rings a trace of $\mathrm{AB}$ oscillations suppression 
at high magnetic fields can be found. In fact, the classical formula for the cyclotron radius $R_{c}=m V / e B$, indicates that the electron paths in a semiconductor will be much more strongly deflected by the magnetic forces than in metals because of the different electron effective masses and Fermi velocities that are both typically two-three orders of magnitude smaller in semiconductors as compared to metals. Oscillations pertaining to relatively high magnetic fields were observed in a semiconductor ring [6] in which tunable barriers were introduced in order to enforce equal transmission in both arms of the ring. One could intuitively expect that the Lorentz force for an asymmetrically connected output lead in a two-terminal ring will lead to an asymmetry of the transfer probability $T(B)$ with respect to the magnetic-field orientation. This is not so because of the microreversibility relation [12] for a two-terminal device $(T(B)=T(-B))$ resulting from the Onsager principle [13]. In this letter we propose an experimental setup where the Lorentz force effect can be unequivocally observed in a quantum ring with two output leads when the current flow in both output terminals is measured. As we show below, in the three-terminal ring, the magnetic field leads to a distinct imbalance of the electron transfer probability from the input terminal to the two output leads. Moreover, the incident-wave-vector resolved probabilities of transfer to the left and right leads show clear correlations as functions of the magnetic field.

Theory. - We solve the time-dependent Schrödinger equation $(i \hbar \mathrm{d} \Psi / \mathrm{d} t=H \Psi)$ for a Hamiltonian of an electron moving on the $(x, y)$-plane in a perpendicular magnetic field $\boldsymbol{B}=(0,0, B), H=(-i \hbar \nabla+e \boldsymbol{A})^{2} / 2 m^{*}$ (we take the GaAs electron band mass $m^{*}=0.067$ ), using the Landau gauge $\boldsymbol{A}=(-B y, 0,0)$. The electron wave function is expanded:

$$
\Psi(x, y, t)=\sum_{n} c_{n}(t) f_{n}(x, y)
$$

in a multicenter basis of displaced Gaussian wave functions

$$
f_{n}(x, y)=\exp \left[-\left(\boldsymbol{r}-\boldsymbol{R}_{n}\right)^{2} / \alpha^{2}+i e B\left(x-X_{n}\right)\left(y+Y_{n}\right) / 2 \hbar\right] / \sqrt{\pi / 2} \alpha
$$

centered around points $\boldsymbol{R}_{n}=\left(X_{n}, Y_{n}\right)$ along the ring and the leads. This approach was previously applied to the magnetic-field-induced Wigner crystallization in a two-dimensional electron gas [14] and in few-electron systems confined in quantum dots [15,16]. The imaginary part of the exponent in (2) guarantees the gauge invariance, i.e. equivalence of all points $\boldsymbol{R}_{n}$ in the magnetic field. Since the electron will be allowed to move only between the centers, their choice defines the model structure geometry. The centers are distributed on a circle of radius $132 \mathrm{~nm}$ forming the quantum ring. The leads are defined by chains of centers oriented parallel to the $y$-axis (see below the insets to figs. 1 and 3). The localization parameter $\alpha$ in (2) is set to $28 \mathrm{~nm}$, which is equivalent to defining a harmonic-oscillator confinement potential in the direction perpendicular to the wires with the confinement energy $\hbar \omega=2.9 \mathrm{meV}$. For the initial condition we take one of the wave functions (2) localized below the ring multiplied by a plane wave $\exp [i q y]$ giving an initial momentum to the wave packet in the direction of the ring. We take $q=0.05 / \mathrm{nm}\left(\hbar^{2} q^{2} / 2 m^{*}=1.42 \mathrm{meV}\right)$ which results in a Gaussian momentum distribution $P(k)=\sqrt{\pi} \sigma \exp \left[-(k-q)^{2} / \sigma^{2}\right]$ with $\sigma=0.0505 / \mathrm{nm}$. The relatively small value of $q$ (in the two-dimensional electron gas a typical value of the Fermi vector is larger by a factor of about 2.5) allows us to reduce the numerical complexity of our calculations by taking a large distance between the centers $(20 \mathrm{~nm})$. For larger $q$ values effects due to the Lorentz force will appear at a higher value of the magnetic field, but otherwise the results should be qualitatively similar. The time dependence of the coefficients $c_{n}(t)$ is calculated with the Askar and Cakmak scheme [17], which for the expansion (1) is given by the system of linear equations $\boldsymbol{S} \boldsymbol{c}(t+\mathrm{d} t)=$ 


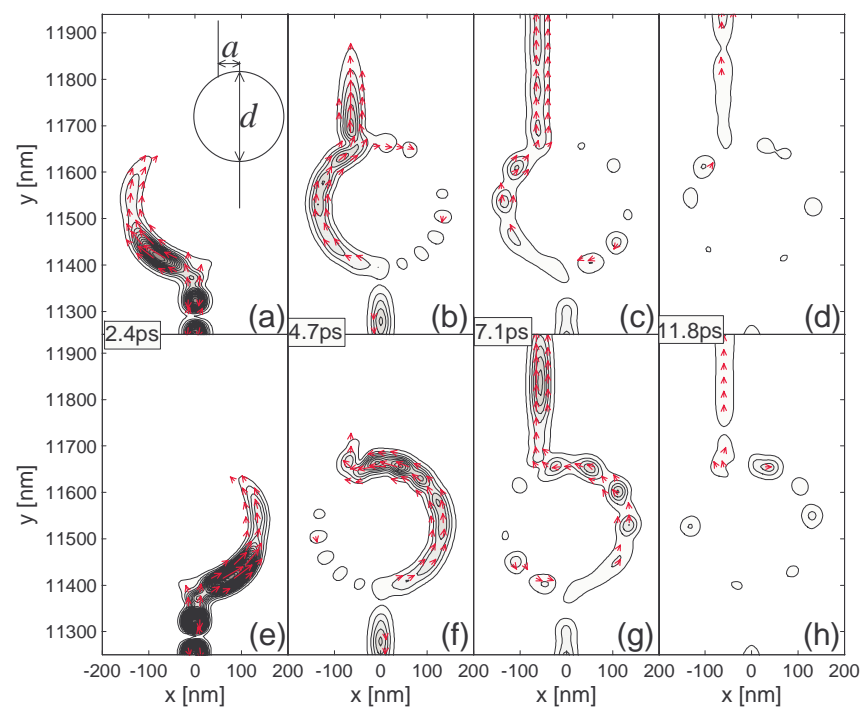

Fig. 1 - (Colour on-line) Charge density contours and probability current vectors for an electron wave packet tunnelling through a two-terminal quantum ring with a single outgoing lead horizontally shifted from the ingoing lead by $a=60 \mathrm{~nm}$ (see the inset in (a)) at $B=0.5 \mathrm{~T}$ (a-d) and $B=-0.5 \mathrm{~T}$ (e-h). Plots $(\mathrm{a}, \mathrm{e}),(\mathrm{b}, \mathrm{f}),(\mathrm{c}, \mathrm{g}),(\mathrm{d}, \mathrm{h})$ correspond to $t=2.4,4.7,7.1$ and $11.8 \mathrm{ps}$, respectively.

$\boldsymbol{S} \boldsymbol{c}(t-\mathrm{d} t)-2 i \mathrm{~d} t \boldsymbol{H} \boldsymbol{c}(t) / \hbar$, with the overlap and Hamiltonian matrix elements given by $\boldsymbol{S}_{k n}=$ $\left\langle f_{k} \mid f_{n}\right\rangle$ and $\boldsymbol{H}_{k n}=\left\langle f_{k}|H| f_{n}\right\rangle$, respectively. In the simulations we take very long leads (the length of the entire computational box is up to $80 \mu \mathrm{m}$ ) as compared to the size of the ring to prevent the wave packet from returning to the ring (due to the finite size of the computational box) before the end of calculations. The crucial features of our modelling are: 1) The wave function cannot change its sign in the direction perpendicular to the wires defined by the choice of centers, so that the calculations are limited to the lowest subband. 2) The integrated flux of the $y$ component of the probability density current $\boldsymbol{j}=\frac{i \hbar}{2 m *}\left(\Psi \nabla \Psi^{*}-\Psi^{*} \nabla \Psi\right)+\frac{e}{m^{*}} \boldsymbol{A} \Psi \Psi^{*}$ equals $\hbar q / m$, so we obtain the same initial condition for all $B$ values. 3) The momentum spectrum for the wave packet travelling through the leads is preserved. In that sense the model of the leads is effectively one-dimensional - the magnetic field cannot rotate the momentum vector for the electron inside the leads, which allows us to discuss the momentum-resolved transmission probability even though the operator $p_{y}=-i \hbar \partial / \partial y$ does not commute with the Hamiltonian.

Results. - Figures 1(a-h) show the charge density and the probability current densities for several moments in time at the magnetic field of $B= \pm 0.5 \mathrm{~T}$ for the outgoing lead attached at a distance $a=60 \mathrm{~nm}$ to the left with respect to the ingoing lead (see the inset to fig. 1(a)). In figs. 1(a,e) we notice that a part of the wave packet is immediately reflected back to the injection lead. The electron packet entering the ring is predominantly injected into the left (for $B=0.5 \mathrm{~T}$ ) or the right (for $B=-0.5 \mathrm{~T}$ ) arm of the ring (see figs. $1(\mathrm{a}, \mathrm{e})$ ) by the Lorentz force. When the packet reaches the point at which the outgoing lead is attached, the Lorentz force directs it out of the ring (left for $B>0$ and right for $B<0$-see figs. $1(\mathrm{~b}, \mathrm{f})$ ). Based on the kinematics of the wave packet ejection to the outgoing lead, one could naively expect that the transfer probability should be larger for $B>0$, since then the electron path has to be deflected by a smaller angle when leaving the ring. The probability to find an electron inside the ring, in the input and output lead is plotted in fig. 2 as a function of time. The packet leaves the 


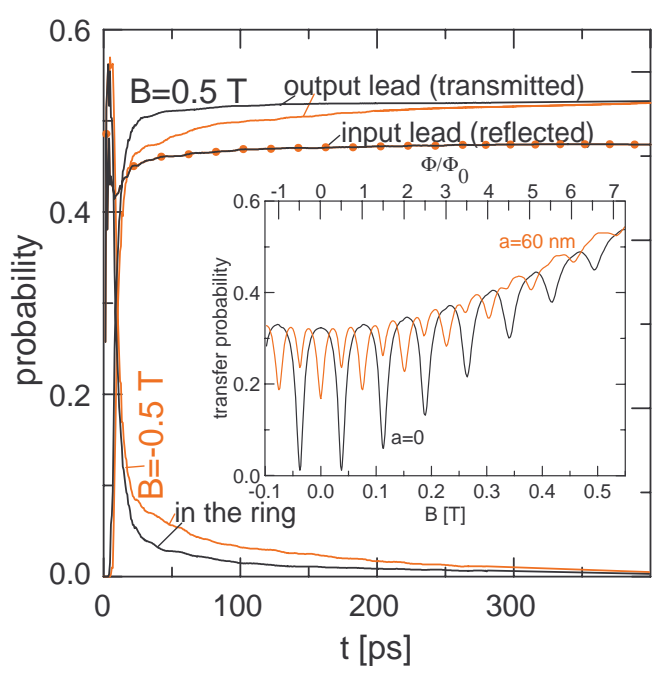

Fig. 2

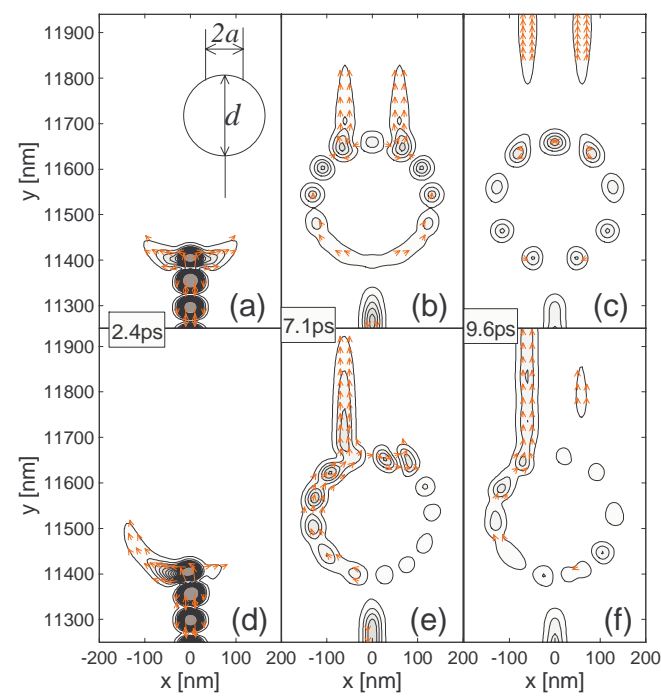

Fig. 3

Fig. 2 - (Colour on-line) The part of the wave packet in the input and output leads and in the ring for $B=0.5$ (black curves) and $-0.5 \mathrm{~T}$ (red curves). Probability to find the electron below the ring for $B=-0.5 \mathrm{~T}$ was marked with red dots. The inset shows the packet transfer probability for $a=60 \mathrm{~nm}$ and for the symmetrical leads $a=0$ as a function of the magnetic field (lower axis) or flux (upper axis).

Fig. 3 - (Colour on-line) Charge density contours and probability current vectors for an electron wave packet tunnelling through a quantum ring with two symmetrically placed outgoing leads spaced in the horizontal direction by $a=60 \mathrm{~nm}$ (see the inset in (a)) at $B=0 \mathrm{~T}$ (a-c) and $B=0.28 \mathrm{~T}$ (d-f). Plots $(\mathrm{a}, \mathrm{d}),(\mathrm{b}, \mathrm{e}),(\mathrm{c}, \mathrm{f})$ correspond to $t=2.4,7.1$ and $9.6 \mathrm{ps}$, respectively.

ring indeed more easily, i.e., faster, for positive values of the field. However, for long times the transmission probability for $B=-0.5 \mathrm{~T}$ eventually reaches the one for $B=0.5 \mathrm{~T}$, and consequently the microreversibility relation $T(B)=T(-B)$ is preserved. Note that the part of the wave packet in the incoming lead for the opposite magnetic-field orientation is identical for all $t$. The gray curve (red on-line) in the inset of fig. 2 shows the transfer probability as a function of the magnetic field for $a=60 \mathrm{~nm}$ which is compared to the case of a symmetrical $(a=0)$ lead configuration (black curve). The single-channel Büttiker $[8,10]$ formula for a symmetric system predicts a strictly periodic $T(B)$-dependence exactly vanishing at the odd multiples of half of the flux quantum $\Phi_{0} / 2,\left(\Phi_{0}=h / e\right.$, for our ring the flux quantum corresponds to $B=0.07557 \mathrm{~T}$ ). This is because, for a symmetric ring, the geometrical phase for paths through the left and right arm is the same and the AB phase for fluxes $(2 n+1) \Phi_{0} / 2$ ( $n$ is an integer) is exactly opposite. The $T(B)$-dependence that we obtain here is not strictly periodic since the present values of the transmission probability at halves of the flux quanta are non-zero and increase with the magnetic field. This is a consequence of the magneticfield-induced imbalance in the packet injection into the left and right arm which prevents the $\mathrm{AB}$ interference at the exit to be completely destructive. In rings with asymmetrically connected leads the Büttiker theory $[5,8,10]$ predicts, for $k$ values at which the geometrical phase shift along the two possible paths is different, conductance minima spaced by halves of the flux quantum and a reduced oscillation amplitude. These features are reproduced in 

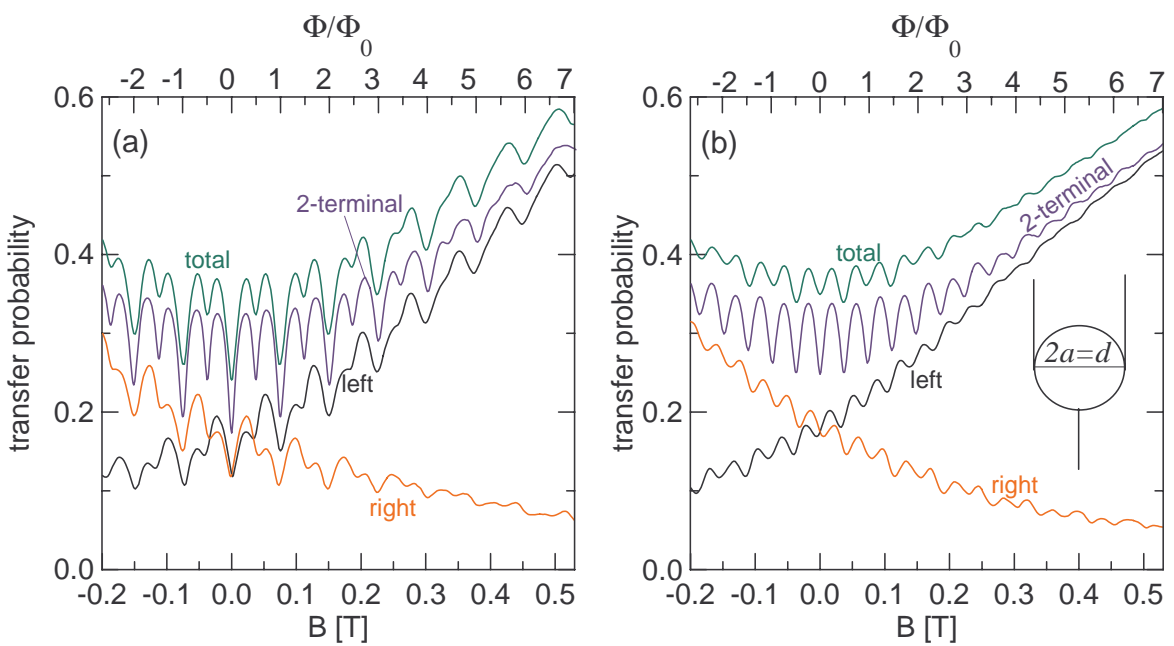

Fig. 4 - (Colour on-line) (a) The packet transfer probability through the left (black curve) and right (red curve) output leads in function of the magnetic field, their sum, i.e. the total transfer probability (green line) and the packet transfer probability for a corresponding (see fig. 1) single output device (blue curve). (b) as (a) only for the output leads attached tangentially to the ring $(a=d / 2=132 \mathrm{~nm}$, see the inset).

our calculations for the wave packet formed by a superposition of plane waves, for which in general, the geometrical phase associated with the two paths is not the same. Minima of the transfer probability at integer multiples of $\Phi_{0} / 2$ for asymmetric rings are usually attributed to interference of the paths performing a whole loop clockwise and counterclockwise around the ring and meeting at the entrance of the ring, which being constructive, increases the chances of backscattering. For closed loops the geometric phases cancel out and for flux $n \Phi_{0} / 2$ so does the relative $\mathrm{AB}$ shift (equal to $2 n \pi$ ), leading to an increased backscattering probability. The overall growth of the transmission probability as a function of the flux is related to the increased guiding of the electron transport due to the Lorentz force.

Let us now consider the device with two output leads, as drawn schematically in the inset of fig. 3(a). We assume that the output leads are placed symmetrically with respect to the incoming lead. In the absence of the magnetic field the packet is transferred equally to both the output leads (see figs. $3(\mathrm{a}-\mathrm{c})$ ). For $B>0$, the current is predominantly directed to the left outgoing lead (figs. 3(d-f)). The black and red (on-line) lines in fig. 4(a) show the packet transfer probability to the left, $T_{l}(B)$, and right, $T_{r}(B)$, outgoing leads, respectively. Due to the symmetrical configuration of the output leads we have $T_{l}(B)=T_{r}(-B)$. The amplitude of the oscillations of both transfer probabilities decreases at high magnetic fields. The shape of the total transfer probability curve, $T_{t}=T_{l}+T_{r}$ (green on-line line) is very similar to the one of a single output device, with one of the output terminals - no matter left or right - removed (blue on-line curve). However, in the three-terminal device the total transfer probability is larger and the amplitude of the oscillations is smaller. Note that at small magnetic fields $\left(|\Phi|\right.$ smaller than $\left.\sim \Phi_{0} / 2\right)$ the transfer probability of the packet is larger for the other lead as expected from the direction of the Lorentz force. This feature is however not reproduced for all $a$. For comparison, the transfer probabilities for a ring with a maximal asymmetry, i.e., for the output leads placed tangentially to the ring, with $a=d / 2$, is plotted in fig. 4(b). In comparison to the case of $a=60 \mathrm{~nm}$ (fig. 4(a)), we see that the slope of the envelope of 

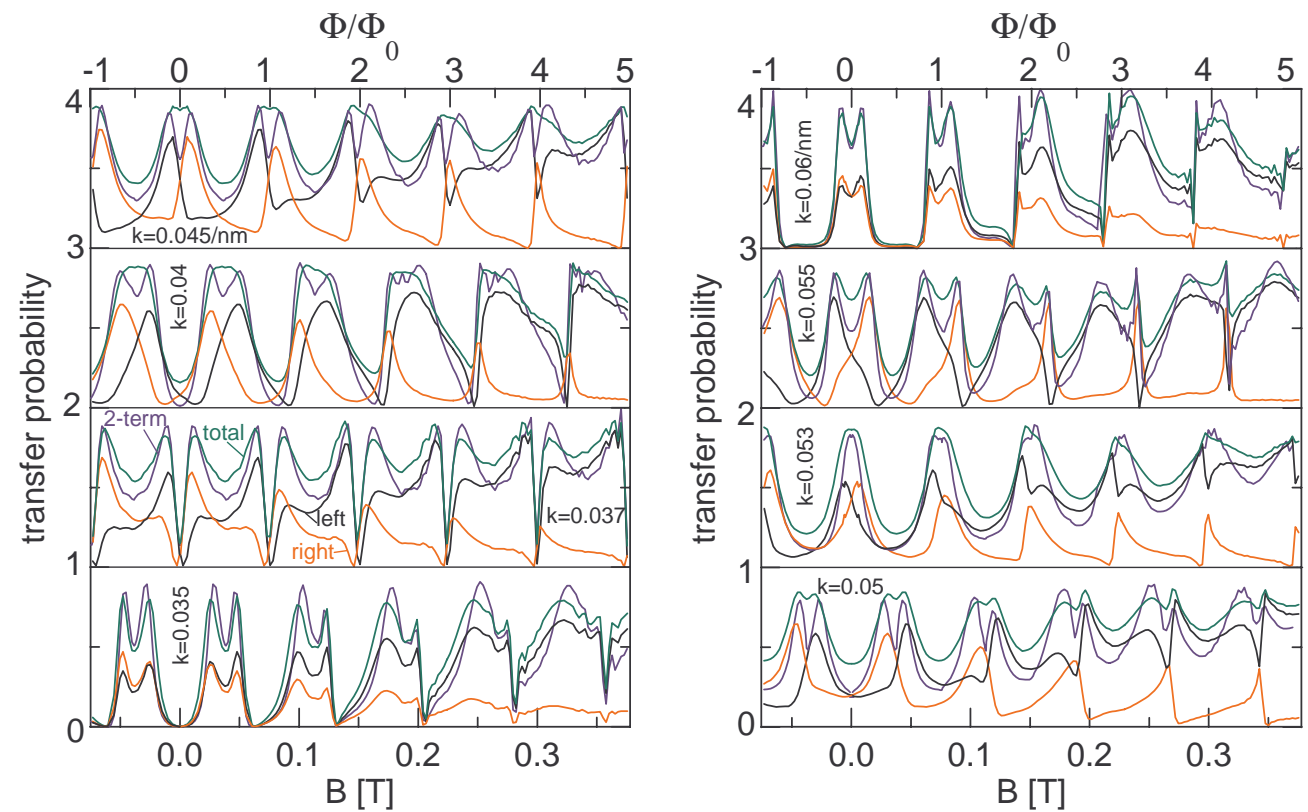

Fig. 5 - (Colour on-line) Transfer probability in function of the magnetic field for the two-output lead with $a=60 \mathrm{~nm}$, for different values of the incident wave vector $k$. Red (black) curves show the transfer probability to the right (left) output leads, green curves show the total transfer probability and the blue ones the transfer probability for one of the output leads removed.

the oscillations for the transfer via left and right leads does not depend on $a$. The decreased amplitude of the oscillations for $a=132 \mathrm{~nm}$ with respect to $a=60 \mathrm{~nm}$ is related to the observed halving of the fundamental $\mathrm{AB}$ oscillations period from $\Phi_{0}$ to $\Phi_{0} / 2$.

Figure 5 shows the incident-momentum-resolved transfer probability as a function of the magnetic field for several $k$ values from the central part of the incident wave packet. The probabilities were calculated using the numerically integrated Fourier transform of the packets at leads axes. In all the plots the total transfer probability $T_{t}$ has a similar $B$-dependence than the probability for a two-terminal device with one of the output leads removed. The amplitude of the oscillations of the two quantities decreases with $B$, similarly as the $k$-averaged signal presented in the packet transfer probability plots of fig. 4. For the lowest value of the wave vector $k=0.035 / \mathrm{nm}$, the transfer probability to the left and right output leads has peaks in the same positions, so that the magnetic field only increases the imbalance of the transfer probability. The double-peak structure of $T_{t}$ with a local minimum at odd multiples of $\Phi_{0} / 2$ gets deteriorated at higher $|B|$ with the height of the peak at the smaller $|B|$ side increasing. Note that a similar dependence is obtained for the maximal $k$ vector value $0.06 / \mathrm{nm}$, only the positions of the $T(B)$ functions extrema are shifted by $\Phi_{0} / 2$ and the peak that is increasing with $|B|$ is the one of the higher $|B|$ side of the central local minimum. For other wave vectors presented in fig. 5 we observe a similar overall increase of the transfer probability as one of the leads is preferred by the Lorentz force. However, we also notice the formation of antilocked extrema of $T_{l}(B)$ and $T_{r}(B)$ at higher magnetic fields. The double-peak structure of the twoterminal device observed for low B in most of the plots can be identified with shifted peaks of $T_{l}$ or $T_{r}$ curves (see the plots for $k=0.037 / \mathrm{nm}$ or $k=0.055 / \mathrm{nm}$, for instance). For $B>0$, as a general rule for the formation of antilocked extrema, we observe that with increasing magnetic 
field the width of the $T_{l}$ peak is increased while the $T_{r}$ peak becomes thinner forming a sharp resonance with an associated dip in the $T_{l}$-dependence on the magnetic field. The antilocked dip-peak structures are spaced by $\Phi_{0}$ so they are still related to the interference of the left and right circulating wave functions, but their depth/height decreases with the magnetic field. AB interference is eventually destroyed when the Lorentz force compels the electrons to follow a single path in the ring - through one arm to the closest lead.

Summary and conclusions. - We show for the first time that the magnetic field introduces, through the Lorentz force, a preferential electron injection into one of the arms of the quantum ring. This imbalance can be measured experimentally in a three-terminal AB device. For a fixed value of the incident wave vector, the magnetic-field dependence of the probabilities of transfer to the symmetrically conected output leads are either correlated with aligned maxima or anticorrelated with maxima of one probability aligned with the minima of the other.

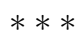

We are grateful to T. IHN and K. ENSSLIN for motivating us to perform this calculation. This work was supported by the Flemish Science Foundation (FWO-Vl) and the Belgian Science Policy, BS is supported by the EC Marie Curie IEF project MEIF-CT-2004-500157.

\section{REFERENCES}

[1] Aharonov Y. and Bohm D., Phys. Rev., 115 (1959) 485.

[2] Webb R. A., Washburn S., Umbach C. P. and Laibowitz R. B., Phys. Rev. Lett., 54 (1985) 2696.

[3] Timp G., Chang A. M., Cunningham J. E., Chang T. Y., Mankiewich P., Behringer R. and Howard R. E., Phys. Rev. Lett., 58 (1987) 2814.

[4] Fuhrer A., Lüscher S., Ihn T., Heinzel T., Ensslin K., Wegscheider W. and Bichler M., Nature (London), 413 (2001) 822.

[5] Pedersen S., Hansen A. E., Kristensen A., Sorensen C. B. and Lindelof P. E., Phys. Rev. B, 61 (2000) 5457.

[6] van der Wiel W. G., Nazarov Yu. V., De Franceschi S., Fujisawa T., Elzerman J. M., Huizeling E. W. G. M., Tarucha S. and Kouwenhoven L. P., Phys. Rev. B, 67 (2003) 033307.

[7] Gefen Y., Imry Y. and Azbel M. Ya., Phys. Rev. Lett., 52 (1984) 129.

[8] Büttiker M., Imry Y. and Azbel M. Ya., Phys. Rev. A, 30 (1984) 1982.

[9] Büttiker M., Imry Y., Landauer R. and Pinhas S., Phys. Rev. B, 31 (1985) 6207.

[10] BütTIKer M., SQUID'85-Superconducting Quantum Interference Devices and their Applications, edited by НАнцвонм H. D. and LübBig H. (Walter de Gruyter, Berlin) 1985, p. 529.

[11] Olariu S. and Popescu I. I., Rev. Mod. Phys., 57 (1985) 339.

[12] Büttiker M., Phys. Rev. Lett., 57 (1986) 1761.

[13] Casimir H. B. G., Rev. Mod. Phys., 17 (1945) 343.

[14] Maki K. and Zotos X., Phys. Rev. B, 28 (1983) 4349.

[15] Szafran B., Peeters F. M., Bednarek S. and Adamowski J., Phys. Rev. B, 69 (2004) 125344 .

[16] Szafran B. and Peeters F. M., Europhys. Lett., 66 (2004) 701.

[17] Askar A. and Cakmak A. C., J. Chem. Phys., 68 (1978) 2794. 